Vol. XXI No $3 \quad 2015$

\title{
RADIO FREQUENCY CO-SITE MANAGEMENT
}

\author{
Iulian BOULEANU, Dorin ALEXANDRESCU, Mircea BORA
}

CICI, Sibiu, Romania,

ibouleanu@gmail.com, adorin6@yahoo.com,mirceabora@yahoo.fr

\begin{abstract}
The radio spectrum is a limited national resource, essential for some governmental applications and increasingly important for a series of non-governmental applications. The allocation of radio resources is done in a centralized manner, designating frequency managers of the defense system structures as local administrators of the resources allotted to the supported echelon. They have a limited number of frequencies they can assign to the emission sources in their area of responsibility. The article addresses the issue of radio spectrum management in the frequency allocation plans when using a large number of emission and reception sources for means of communication and non-communication in a small area. Locating several emission sources in the same site leads to different types of disturbing signals: emissions outside the bandwidth, harmonics and intermodulation. The article categorizes and describes these sources, presents the results of measurements distinguishing them, as well as the results of implementing some protective measures. Finally, the authors suggest a software solution for the local distribution of frequency resources.
\end{abstract}

\section{Keywords: interference, intermodulation, co-site, allocation, radiofrequency}

\section{Introduction}

The electromagnetic spectrum is composed of an infinity of electromagnetic waves. Though infinite, this spectrum can not provide unlimited resources to any user because there are a number of limitations which make the frequency band available for applications that use electromagnetic waves to be confined to a subdomain of the electromagnetic spectrum, known as the radio waves domain. A first limitation is imposed by the technology that we have. A second limitation is related to the specific nature of wave propagation: the smaller the wavelength, the greater the signal attenuation with respect to the distance, which leads to reduced terrestrial link distances. In terms of frequency distribution, the most important limitation is imposed by the effect of interferences. In their propagation from the transmitter to the receiver, waves move using the electromagnetic field. The electromagnetic field radiates in the space surrounding the antennas often infesting spatial domains the radio service does not need. The available technologies allow only to a very small extent the guidance of the electromagnetic field from the transmitter to the receiver and therefore, in every point of the electromagnetic environment, electromagnetic waves are overlapping, not only of different frequencies, but even of the same frequency, leading to reciprocal disruption of some services.

Within its borders, every state is the owner of the entire electromagnetic spectrum. Due to the nature of wave propagation, which does not respect geographical boundaries, access to the spectrum is regulated by national, regional and global decisions. The area of the electromagnetic spectrum covered by regulations is only that of radio frequencies. 
The electromagnetic spectrum between infrared waves and gamma waves and the frequency bands up to $8.75 \mathrm{kHz}$ are not subject to national and international regulations.

Controlling the phenomenon of interference is approached by a process which takes place in two stages.

The first step is to regulate access to the national spectrum so that each user has a set of parameters that specify the spatial, temporal, spectral, and technological characteristics of the emission. These parameters are set by the national authority managing the frequencies so that users who have obtained the right to emit do not cause harmful interference to other active users on these emission parameter sets.

Regulating access to the spectrum implies a process of allocation, allotment and finally assignment of radio frequencies.

The second stage of the process covered by this article consists of creating conditions that enable several transmitters and receivers to operate in a small area.

Although, by the process of assigning frequencies for co-located users, each equipment has been distributed parameters so that the spectral components of emitting systems do not overlap, due to spurious signals produced by each emitting equipment (out-of-band signals, harmonics, etc.) and the simultaneous operation of several emitting sources (intermodulation), co-located receivers can receive harmful emissions on frequencies other than those assigned by the national authority. This second stage involves the identification of sets of emission characteristics which, assigned to a group of co-locate transmitters and receivers, do not cause perturbations on the frequencies assigned to the receivers.

\section{Local sources of perturbations}

According to ITU - Radio Regulation (International Telecommunication Union), interference represents "... the effect of unwanted energy due to one or a combination of emissions, radiations, or inductions upon reception in a radiocommunication system, manifested by any performance degradation, misinterpretation, or loss of information which could be extracted in the absence of such unwanted energy". [1]

During the processing of the emitted signal, due to the nature of signal modulation and the nonlinear characteristics of some levels in the emission chain, in the transmitter's antenna, in addition to the desired signal, there arrive numerous other signals (Fig. 1).

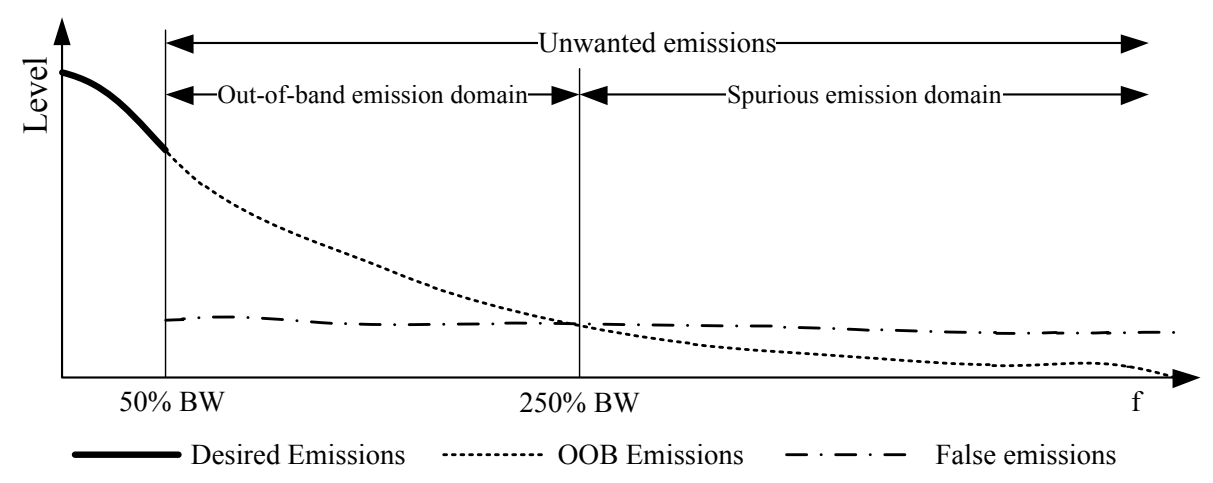

Figure 1: Unwanted emissions [2]

ITU-RR defines these unwanted emissions as being composed of out-of-band emissions and spurious emissions. Out-ofband emission represents emission on a frequency or frequencies immediately outside the necessary bandwidth which results from the modulation process. Spurious emission represents emission on a frequency or frequencies which are outside the necessary bandwidth and the level of which may be reduced without affecting the corresponding transmission of information. 
Spurious emissions include harmonic emissions, parasitic emissions, intermodulation products and frequency conversion products [1].

The effect of interferences due to unwanted emissions is felt especially when multiple radios are co-located. Although radio equipment manufacturers are working hard to reduce unwanted emissions, they succeeded only to mitigate these emissions and not to totally eliminate them. Significant reduction of false emissions with values of at least $60 \mathrm{~dB}$ (typical $90 \mathrm{~dB}$ ) causes the unwanted effect of interference from these signals to be felt only in the vicinity of emission sources.

\subsection{Adjacent channel interference}

Out-of-band emissions (OOB) have the highest levels of interference. To avoid adjacent channel interferences, no adjacent frequency channels are assigned in the same service area [3]. From Fig. 1 there can be deduced (1).

$\mathrm{f} \neq\left(\mathrm{f}_{\mathrm{a}}-3 \cdot \mathrm{BW} \ldots \mathrm{f}_{\mathrm{a}}+3 \cdot \mathrm{BW}\right)$

where $f_{a}$ is the central frequency assigned to a channel with a bandwidth of $\mathrm{BW}$, and $f$ is the central frequency of another channel that can be assigned. Therefore in the assignment process there will not be assigned six adjacent channels (three left and three right of the assigned frequency).

\subsection{Harmonics Interference}

In the category of false emissions, the frequency manager must consider the harmonics of each transmitter and the products of intermodulation (PIM) occurring at the output of several simultaneous broadcasting transmitters. Harmonics occur due to processing the signal in the nonlinear floors. They form as exact multiples of the emission frequency. Around each harmonic there is a replica of the RF signal. The harmonics level decreases with the increase of the harmonics order and increases with the increase of the transmission power.

To remove interferences that may occur due to harmonics, when assigning a new frequency $f$, the frequency manager shall check if the following set of relationships is met:

$\mathrm{f} \neq \mathrm{nf}_{\mathrm{a} 1} ; \mathrm{f} \neq \mathrm{nf}_{\mathrm{a} 2} ; \ldots \mathrm{f} \neq \mathrm{nf}_{\mathrm{am}} ;$

where $f_{a 1}, f_{a 2}, \ldots f_{a m}$ are already assigned frequencies, and $n$ is a positive integer representing the number of the harmonic. Common values for $n$ are 2 or 3 for emission sources having emitting powers up to several watts, which can grow up to order 7 for emission sources of several kilowatt.

\subsection{Intermodulation interference}

PIM occur when coupling two or more transmitters. Useful and spurious signals emitted by each transmitter are induced in the antennas of other co-located transmitters and end up in nonlinear output floors where they are composed with the signals produced by each transmitter. When there are more transmitters, then more PIM are obtained. The exact level of intermodulation products depends, as in the case of harmonics, on a series of equipment parameters, but it follows a Gaussian pattern. This pattern can be distinguished in Figure 2, which presents the harmonic components and the PIM resulting when two transmitters $(41.75 \mathrm{MHz}$ and $42.25 \mathrm{MHz}$ ) are working together. 


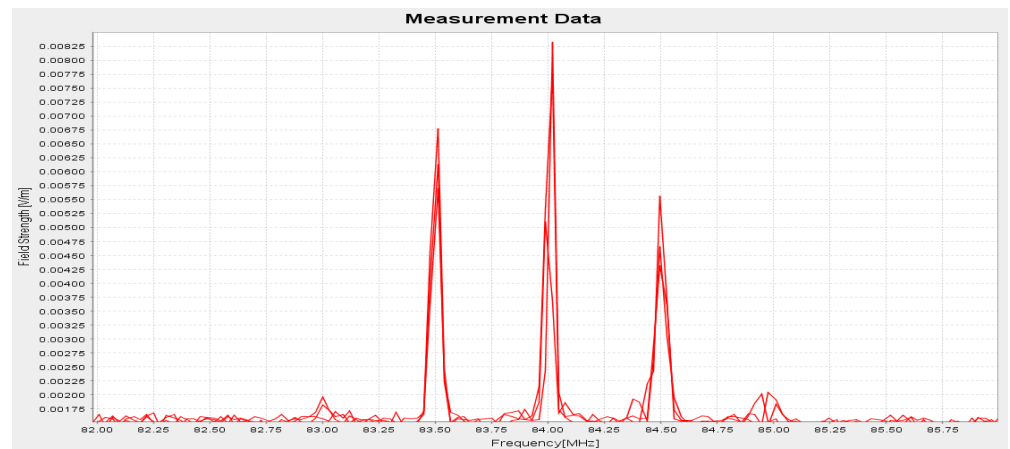

Figure 2: Amplitude pattern of intermodulation products and its harmonic component

$S$

Components of $83.5 \mathrm{MHz}$ and $84.5 \mathrm{MHz}$ are harmonics of the emitted frequencies. The other ingredients are PIM. The PIM's frequency value resulting from harmonic components have higher levels than those of harmonics and PIM's frequency further from the central value levels are becoming smaller.

Equation (3) describes the relationship between the frequencies of $m$ co-located emission sources and the frequencies of the intermodulation products $\left(f_{P I M}\right)$ obtained:

$\mathrm{f}_{\mathrm{PIM}}= \pm \mathrm{nf}_{\mathrm{a} 1} \pm \mathrm{xf}_{\mathrm{a} 2} \pm \ldots \pm \mathrm{yf}_{\mathrm{a} i} \pm \ldots \pm \mathrm{zf}_{\mathrm{am}}$ where $f_{a i}$ is the frequency of transmitter $i$ of the $m$ transmitters and $n, x, y$ and $z$ are the harmonics of the assigned frequencies. The order of PIM is obtained by summing the orders of component harmonics.

The most dangerous PIM are those whose frequency is in the operating band of the emission source (eg. those of the 3 order)[3]. As the order of intermodulation products increases and the resulting $f_{P I M}$ departs from the assigned frequencies, the level of PIM decreases. The number of harmonics considered for forming intermodulation products depends on the output power and usually can range up to 7 .

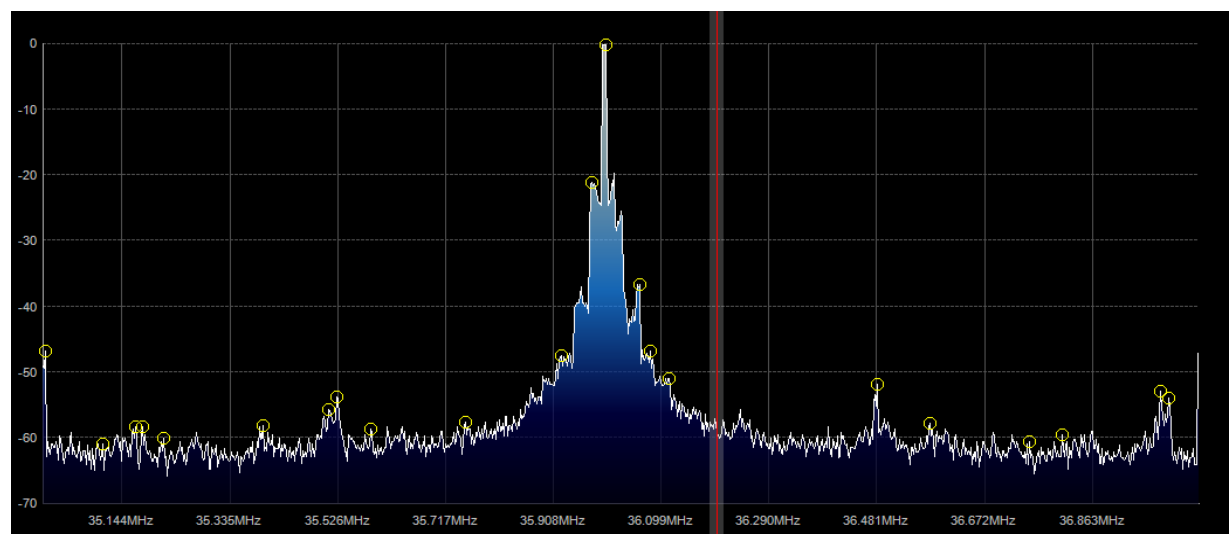

Figure 3: $O O B$ and useful signal emissions for a STANAG 5042 source type

\section{Evaluation of the level of spurious emissions}

To highlight the relationship between the levels of useful emissions and spurious emissions, two experiments were conducted in which the two emission levels were compared to emission sources compliant to STANAG 5042 [4].

\subsection{Evaluation of $\mathrm{OOB}$ emission levels}

To assess the OOB emission levels, a real- time analyzer of the Software Define Radio type was used, consisting of a dongle RTL2832U + E4000 [5] along with the SDRSharp V1.0.0.1163 application [6]. The emission source, located at a distance of $10 \mathrm{~m}$, issued an FSK signal with $\mathrm{BW}=25$ $\mathrm{kHz}$ on the frequency of $36 \mathrm{MHz}$. The measurement result is shown in Figure 3. The normed value of the received signal level is $0 \mathrm{dBm}$ for frequencies of the 25 
$\mathrm{kHz}$ channel. The signal level in the two adjacent channels is reduced with $20 \mathrm{~dB}$. The signal level of the next two adjacent channels is again reduced with $20 \mathrm{~dB}$. In fact, the significant amounts of the emission signal level, greater than $-50 \mathrm{~dB}$, are located in a bandwidth of almost $200 \mathrm{kHz}$ around the center emission frequency. Thus, when assigning frequencies, the frequency manager should not use the 6 channels adjacent to the assigned channel.

\subsection{Evaluation of the harmonic level and intermodulation level}

The level of the harmonic components is a parameter which we can deduce from the information contained in the data sheet equipment catalog. Their level is related to the carrier level and is determined by the characteristics of the hardware components of the equipment. The frequency manager (or the operator) can act in order to reduce them.

From Figure 2 it is inferred that the PIM levels are comparable with those of the harmonic components. In practice, these levels depend on the difference between the carrier frequencies and the distance between the antennas of the transmitters (the smaller the difference or distance, the higher the intermodulation product levels), on the characteristics of the environment (strongly or weakly reflexive), and on the directivity of the antennas [3]. These are the parameters over which the manager or the operator can act in order to reduce intermodulation products.

Since harmonic components and intermodulation products levels are only 60 $\mathrm{dBm}$ to $90 \mathrm{dBm}$ lower than carrier levels, for receivers in the same location they are powerful sources of disturbance as the emitted signal can reach the receiver at values close to its sensitivity value (i.e. $110 \mathrm{dBm}$ ) while the harmonic components and the intermodulation products can reach levels of approximately -30 to $-60 \mathrm{dBm}$ at the point of reception.

4. Software administration of the frequencies assigned to a particular site
In a site where it is necessary to install several emission sources and several receivers, the frequency manager has a number of frequencies which he has to assign so as to avoid the occurrence of harmonic components and intermodulation products of frequencies from the same set. To perform this operation in optimal time, the frequency manager must use software solutions that take into account all possible combinations resulting by applying the above relations.

The software application $S o r t F q$ has been developed by the authors for this purpose. Using $\operatorname{SortFq}$, the frequency manager can divide the set of available frequencies in several sub-sets, so that any frequency within each newly created sub-set shall not be the harmonic component or intermodulation product of any combination of the other frequencies of the sub-set.

The application can manage a number of up to 300 frequencies, which it can divide into sub-sets of maximum 50 frequencies.

The size of the initial frequency set and the maximum dimension of each sub-set of frequencies will be established using the Settings/Set Size menu. The order of the harmonics considered during frequency sorting is also specified here (Figure $4 a$ ).

In the initial set of frequencies (Frequencies/Initial Set) there will be introduced all frequencies from the frequency allocation plan remaining available for the targeted site (Figure $4 b$ ).

Grouping the frequencies in sub-sets is done in the Frequencies/Grouping Frequencies menu (Figure 4c). To launch the process of frequency selection, the frequency manager will select a frequency (any frequency) of the initial set and introduce it in the first subset. Further, the application will test each frequency in the initial set with equation (3) to determine if it is a harmonic component or intermodulation product. Frequencies which are not a harmonic component or intermodulation product are assigned to this sub-set and are removed from the initial set. 
For the following sub-sets, the frequency manager proceeds as for the first set, the frequency sorting considering each time a shorter list of frequencies.

Each set of frequencies thus obtained is free from interference due to other users of the site. The frequencies in each set can be distributed to transmission and reception equipment grouped into one site. The distance between sites with different assignment lists must be determined so that harmonic component and intermodulation product levels received from neighboring sites fall below the receiver's sensitivity threshold. This can be achieved for a propagation attenuation of about $60 \mathrm{dBm}$ to $90 \mathrm{dBm}$.

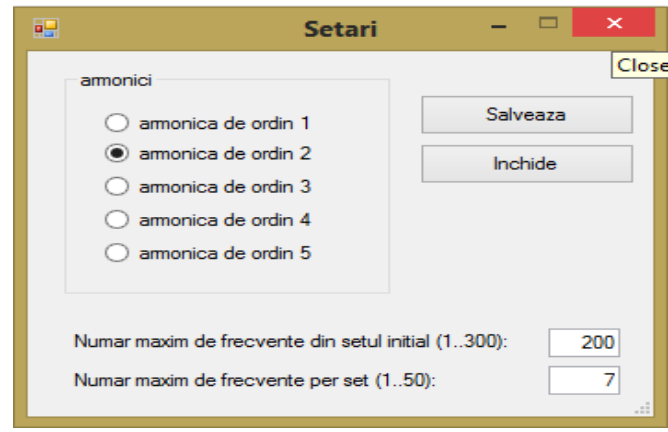

a) Settings menu

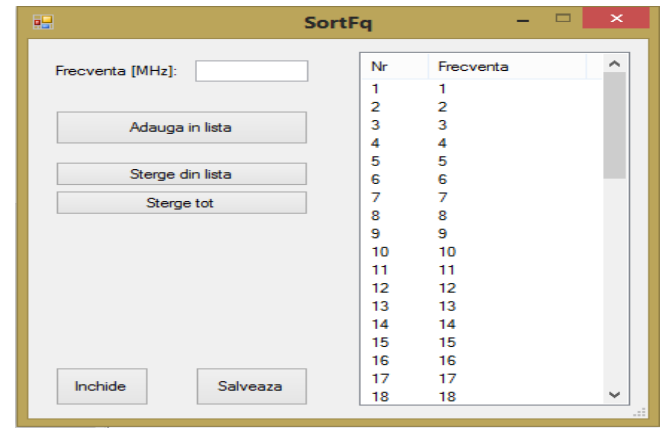

b) Initial Set menu

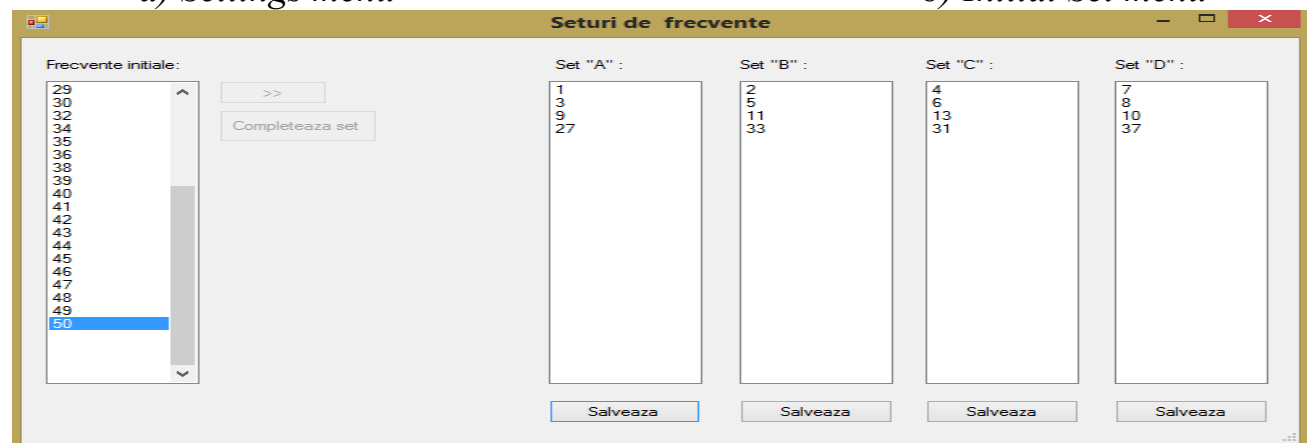

c) Frequency Sorting menu

Figure 4. Menus of the SortFq application

\section{Conclusions}

The article addresses the issue of radio spectrum management in the frequency allocation plans when using a large number of emission and reception sources for means of communication and noncommunication in a small area.
The article describes sources of in-site interferences, presents the results of measurements distinguishing them, as well as the results of implementing some protective measures. Finally, the authors present a software solution for the local distribution of frequency resources.

\section{References}

[1] ITU, ITU- Radio Regulation - Articles, Vol. 1, 2012

[2] ECC (02)05, Unwanted Emissions, ECC Recommendation, 2002

[3] Bechet P., Munteanu R., Bouleanu I., Munteanu M., Mitran R., Electromagnetic Compatibility Average Radio Communications, București, Ed. Academiei Române, 2010

[4] NATO, STANAG 4204 Technical Standards for Single Channel VHF Radio Equipment, Edition 3, 2008

[5] http://sdr.osmocom.org/trac/wiki/rtl-sdr, accessed on 03.03.2015

[6] http://www.joshknows.com/gnuradio_port, accessed on 03.03.2015 